\title{
Occurrence of De Quervain's Thyroiditis after Resolution of Hypercortisolism following Pasireotide Treatment for Cushing's Disease and Surgery for an Adrenocortical Adenoma: Report of Two Cases
}

\author{
Zoe A. Efstathiadou Albana Sykja Panagiotis Anagnostis Athanasios Panagiotou \\ Marina Kita \\ Department of Endocrinology, 'Hippokration' General Hospital of Thessaloniki, Thessaloniki, Greece
}

\section{What Is Known about This Topic?}

- New onset and exacerbation of autoimmune thyroid disease have been described after successful treatment of endogenous hypercortisolism.

\section{What Does This Case Report Add?}

- It seems that correction of endogenous hypercortisolism also predisposes to thyrolytic processes not traditionally related to thyroid autoimmunity, especially while the hypothalamus-pituitary-adrenal axis is still suppressed.

\section{Key Words}

De Quervain's thyroiditis · Cushing's disease ·

Cortisol-secreting adrenal adenoma - Pasireotide .

Endogenous hypercortisolism - Glucocorticoid withdrawal syndrome

\section{Abstract \\ Objective: An increased prevalence of thyroid autoimmu- nity has been observed after successful treatment of Cush- ing's syndrome. On the other hand, De Quervain's thyroiditis (DQT), in which autoimmunity is not a pathogenetic contrib- utor, has not been reported during recovery from Cushing's syndrome. We describe 2 female patients with DQT coincid- ing with the resolution of hypercortisolism after treatment}

of Cushing's syndrome/disease. Methods: The first patient had been diagnosed with Cushing's disease due to a corticotroph pituitary microadenoma, declined neurosurgery, and was receiving pharmacological treatment with pasireotide. Her hypercortisolism was optimally controlled with a minimum dose. The second patient had undergone unilateral adrenalectomy due to a cortisol-secreting adenoma and was on tapering doses of hydrocortisone due to a suppressed corticotroph axis. Both patients presented with clinical, functional, and imaging features of DQT at a time when their endogenous glucocorticoid levels were very low. Results: Oral glucocorticoid treatment was administered in both cases, resulting in prompt recovery. Conclusions: The incidence of DQT following the resolution of hypercortisolism, either medical or surgical, has not been previously described. The

\begin{tabular}{ll}
\hline KARGER & $\begin{array}{l}\text { (c) 2014 European Thyroid Association } \\
\text { Published by S. Karger AG, Basel } \\
2235-0640 / 14 / 0031-0069 \$ 39.50 / 0\end{array}$ \\
$\begin{array}{l}\text { E-Mail karger@karger.com } \\
\text { www.karger.com/etj }\end{array}$ &
\end{tabular}

Zoe A. Efstathiadou, $\mathrm{PhD}$

Department of Endocrinology, 'Hippokration' General Hospital of Thessaloniki Konstantinoupoleos 49 GR-54642 Thessaloniki (Greece)

E-Mail zefsta@endo.gr 
exact pathogenetic mechanism can only be speculated on. Perhaps the relative or absolute glucocorticoid deficiency after effective treatment of hypercortisolism alters immunologic responses and renders patients more vulnerable to thyrolytic processes.

C 2014 European Thyroid Association Published by S. Karger AG, Basel

\section{Introduction}

Exacerbation and increased prevalence of autoimmune thyroid dysfunction after successful treatment of Cushing's syndrome have been described in small patient series in both ACTH-dependent [1] and ACTH-independent $[2,3]$ clinical settings. These findings seem to be justified by the recognized inhibitory effects of glucocorticoids on immune function [4]. Indeed, the state of immunosuppression created by glucocorticoid excess is associated with improvement in autoimmune diseases. However, upon remission of hypercortisolism, exacerbation of preexisting or de novo occurrence of autoimmune or allergic disorders has been reported [5].

Contrary to Hashimoto's thyroiditis, in subacute or De Quervain's thyroiditis (DQT) autoimmunity does not appear to play a primary pathogenetic role [6]. The disease presents with characteristics of viral infections, such as a prodrome with myalgias, malaise, and fatigue. The thyroid injury is attributed to cytolytic T-cell recognition of viral and cell antigens presented in an appropriate complex [6]. To date, no case of DQT occurring after effective treatment of Cushing's syndrome has been reported.

We describe 2 cases of DQT coinciding with the resolution of hypercortisolism after treatment of Cushing's syndrome/disease.

\section{Case 1}

A 41-year-old female who had been diagnosed with Cushing's disease 5 years earlier, but had refused neurosurgery on several occasions throughout follow-up, consented to administration of pasireotide (SOM230).

Her medical history was positive for several components of the metabolic syndrome, i.e. diabetes mellitus, arterial hypertension, dyslipidemia - all adequately controlled with oral medication (metformin and sitagliptin, telmisartan, and simvastatin). Subclinical hypothyroidism had also been diagnosed a couple of years before, with negative thyroid autoantibodies, and the patient was on L-thyroxin treatment $(62 \mu \mathrm{g} /$ day $)$.
After clinical and laboratory evaluations, she was started on pasireotide at an initial dose of $600 \mu \mathrm{g}$ t.i.d. Twenty days after drug initiation, the dose was reduced to $300 \mu \mathrm{g}$ t.i.d. owing to difficulties controlling blood glucose levels despite the increased dose of her current antidiabetic medication (metformin and sitagliptin) and the addition of gliclazide at maximum doses to her regimen. The patient responded appropriately to pasireotide treatment, with a marked reduction of plasma cortisol and ACTH and 24-hour urine cortisol levels (fig. 1).

In the third month of successful treatment, she experienced a febrile state accompanied by malaise and severe neck pain. At physical examination she looked unwell, with a body temperature of $37.5^{\circ} \mathrm{C}$. The thyroid was tender at palpation and she had an increased pulse rate at 100 beats $/ \mathrm{min}$.

Laboratory tests revealed normal white blood cell count with elevated ESR $(110 \mathrm{~mm} / \mathrm{h})$ and thyrotoxicosis $[\mathrm{TSH}=0.065 \mathrm{mIU} / \mathrm{l}$ (reference 0.4-4), FT4 $=25.74 \mathrm{pmol} / \mathrm{l}$ (reference 10.3-22.7); TSH at the initiation of pasireotide $=3.78 \mathrm{mIU} / \mathrm{ll}$. Serum anti-thyroid peroxidase and anti-thyroglobulin antibodies remained undetectable. At the same time, her morning plasma cortisol (8.00 a.m.) was $71.7 \mathrm{nmol} / \mathrm{l}$ (reference 110-607) and her UFC was $11 \mathrm{nmol} / 24 \mathrm{~h}$ (reference 11-138).

The patient underwent technetium thyroid scintigraphy, which showed a complete absence of thyroid uptake, and a thyroid ultrasound that revealed a diffusely hypoechogenic gland with low blood flow. Consequently, the diagnosis of DQT was confirmed and pasireotide and L-thyroxin were temporarily discontinued.

The symptoms did not resolve after a short course of NSAIDs, and after 3 days the patient was started on methylprednisolone (32 $\mathrm{mg} /$ day), resulting in dramatic clinical improvement. The dose was tapered gradually over 1 month, and 2 weeks after cessation pasireotide was reinitiated. The dose of pasireotide was further adjusted to $300 \mu \mathrm{g} / \mathrm{day}$ after 2 months because the patient experienced glucocorticoid withdrawal symptoms. After 3 months of treatment at this dose, the patient presented with erythema nodosum. A subsequent workup with an antistreptolysin-O titer to exclude streptococcal infection, a chest radiograph to exclude signs of pulmonary sarcoidosis, tuberculosis, or fungal infection, and skin testing for tuberculosis were negative for the abovementioned situations.

\section{Case 2}

A 50-year-old woman with Cushing's syndrome was subjected to unilateral left adrenalectomy due to a cortisol-secreting adrenocortical adenoma. After surgery, she was placed on glucocorticoid substitution with instructions to taper the dose and discontinue the treatment after 2 months. However, the patient had severe symptoms and signs of hypocortisolism with nausea, anorexia, diffuse joint pain, and hypotension. At that time, continuation of glucocorticoid substitution with hydrocortisone (25 $\mathrm{mg} /$ day) was decided upon. In the fifth month postsurgery, while the hydrocortisone dose was tapered to $20 \mathrm{mg} /$ day, the patient developed left-sided neck pain irradiating to the jaw and ear, accompanied by fever of up to $38.5^{\circ} \mathrm{C}$ and malaise. Physical examination showed marked sensitivity at thyroid palpation, tachycardia, and tremor. Further workup revealed mild thyrotoxicosis $[\mathrm{TSH}=0.01 \mathrm{mIU} / \mathrm{l}, \mathrm{FT} 4=20.6 \mathrm{pmol} / \mathrm{l}$, and TT3 $=2.49 \mathrm{pmol} / \mathrm{l}$ (reference 1.08-2.62)], increased ESR $(45 \mathrm{~mm} / \mathrm{h})$, and negative 


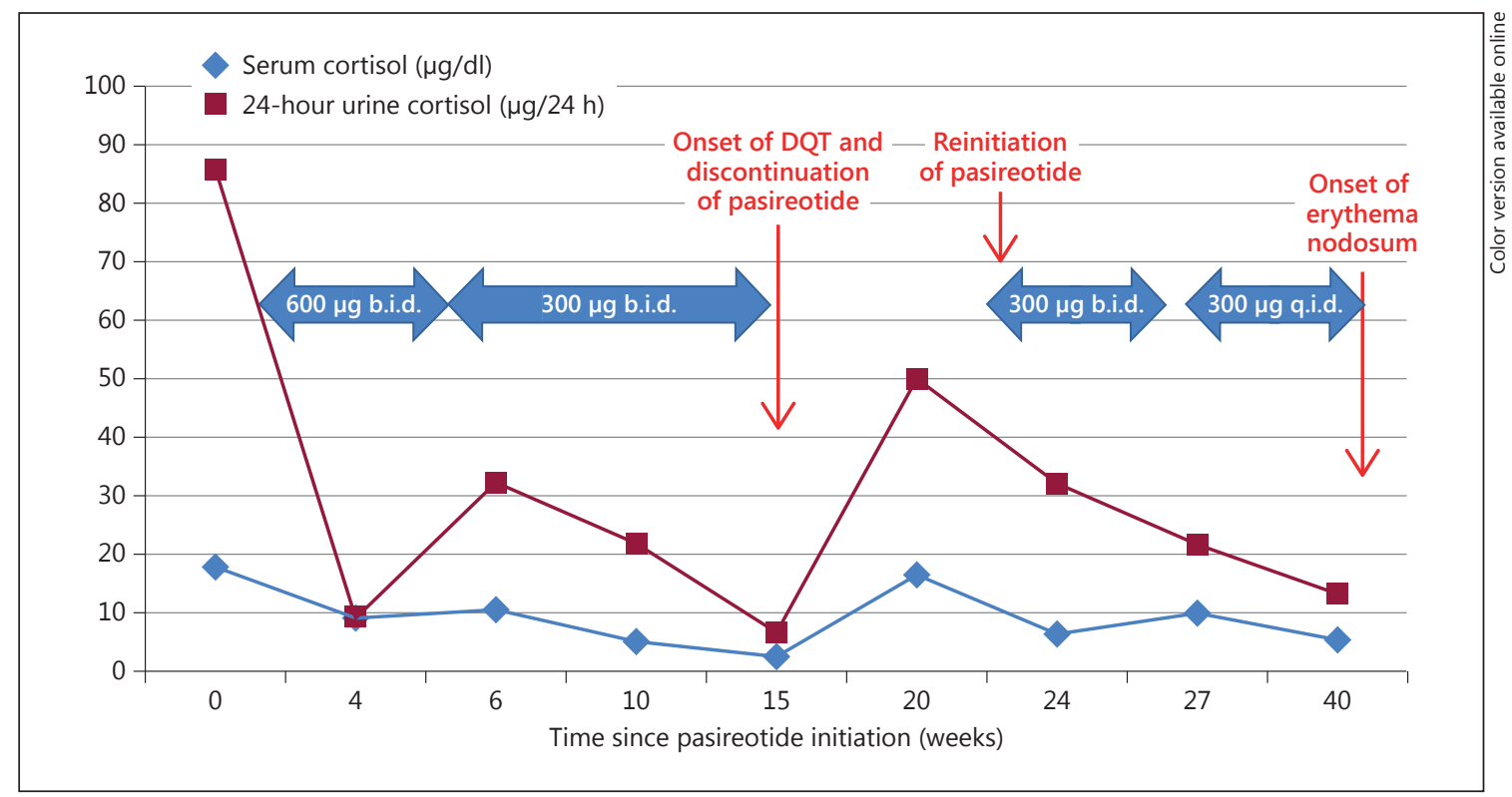

Fig. 1. Oscillations of morning plasma and 24-hour urine cortisol levels during pasireotide treatment and occurrence of DQT and erythema nodosum in case 1 . The periods of pasireotide treatment and the respective doses are depicted within the double-headed arrows.

thyroid autoantibodies. Her morning plasma cortisol (8.00 a.m.) - before her morning substitution dose - at that time was still very low (13.8 nmol/l). A technetium thyroid scan was done, and thyroid uptake was absent. The hydrocortisone was discontinued and the patient was started on prednisolone $(30 \mathrm{mg} /$ day $)$, which resulted in immediate relief of symptoms. With every attempt to taper the dose, she experienced signs of relapse. She returned to the substitution glucocorticoid dose after 6 months.

Informed consent was obtained from both patients for presentation of the data.

\section{Discussion}

We described 2 cases of endogenous hypercortisolism in which subacute DQT occurred after successful treatment. There are 2 major differences between the cases regarding the etiology of and therapeutic approach to Cushing's syndrome. In the first case, a corticotroph pituitary adenoma was causing Cushing's disease, which was pharmacologically treated with the somatostatin receptor analogue pasireotide. In the second case, a cortisol-secreting adrenal adenoma was diagnosed and surgically excised. On the other hand, a remarkable similarity between the cases was that the timing of the appearance of DQT, which is a corticosteroid-responsive condition, coincided with the fall of endogenous cortisol to very low levels, i.e. below the range of normality, in both cases.

The most probable etiology for DQT is a viral illness [6]. This hypothesis is supported by the fact that episodes of DQT often follow upper respiratory tract infections and are associated with falling viral titers of several viruses, including influenza, adenovirus, mumps, and Coxsackie virus, during recovery. DQT is not associated with autoimmune thyroiditis, and even though a transient presence of thyroid autoantibodies might be noted in the acute phase of the disease, this has been attributed to a virally induced autoimmune response and has not been implicated in the pathophysiologic process. In a similar manner, erythema nodosum, which further complicated the treatment of the first patient, is considered to be an immunologic response to a variety of causes - primarily infections, drugs, and connective tissue disease - with a considerable proportion of cases with an unknown etiology [7].

The incidence of DQT upon successful treatment of Cushing's syndrome has not been previously described in the literature. On the other hand, there exist a number of reports of increased prevalence or exacerbation of thyroid autoimmunity after successful treatment of Cushing's syndrome [2] or disease [1]. 
At present, the existence of an underlying pathophysiological mechanism of DQT occurrence after treatment of Cushing's syndrome can only be hypothesized. DQT in the 2 presented cases could merely represent a fortuitous event. Alternatively, it can be speculated that the relative or absolute glucocorticoid deficiency after effective treatment of hypercortisolism alters immunologic responses and renders patients more vulnerable to thyrolytic processes. The thyroid injury in DQT is thought to result from the cytolytic T-cell recognition of viral or cell antigens present in an appropriate complex [6]. This hypothesis is reinforced by the appearance of a second disorder with a similar underlying cell-mediated immune response - erythema nodosum - in the first patient in a short time frame. However, an either direct or indirect effect of pasireotide, through its actions on the immune system, on the pathogenesis of DQT in the first patient cannot be entirely excluded.

It is proven that conditions associated with significant alterations in glucocorticoid levels, either causing their increase as happens at the initiation of stress or causing their decrease as is the case upon cessation of chronic stress or pregnancy, could affect the susceptibility to viruses or the course of infections, as well as autoimmune or atopic diseases, through modulation of the Th1/Th2 balance [8]. In our 2 cases, the fall in glucocorticoid levels was the result of successful treatment of endogenous hypercortisolism of 2 different etiologies.

Furthermore, in experimental models it has been shown that withdrawal of adrenal glucocorticoids produces alterations in the thymocyte selection processes, possibly affecting the diversity of the functional T-cell repertoire and the generation of potentially self-reactive cells [9].

Another possible mechanism via which glucocorticoid withdrawal could affect the integrity of thyroid cells is through effects on the vasculature and most importantly on vascular smooth muscle cells and endothelium.

In conclusion, the occurrence of DQT could coincide with effective treatment of endogenous hypercortisolism, especially at times when glucocorticoid withdrawal syndrome is present. Awareness of this possibility could lead to prompt recognition of the situation, but further research is needed to elucidate the underling pathogenetic mechanism.

\section{Disclosure Statement}

Z.A.E., A.S., and M.K. participated as investigators in a phase IIIb study of pasireotide in patients with Cushing's syndrome (Seascape), sponsored by Novartis. P.A. and A.P. have nothing to disclose.

\section{References}

$>1$ Colao A, Pivonello R, Faggiano A Filippella M, Ferone D, Di Somma C, Cerbone G, Marzullo P, Fenzi G, Lombardi G: Increased prevalence of thyroid autoimmunity in patients successfully treated for Cushing's disease. Clin Endocrinol 2000;53:13.

-2 Takasu N, Komiya I, Nagasawa Y, Asawa T, Yamada T: Exacerbation of autoimmune thyroid dysfunction after unilateral adrenalectomy in patients with Cushing's syndrome due to an adrenocortical adenoma. $\mathrm{N}$ Engl J Med 1990;332:1708.
Kimura H, Sato K, Nishimaki M, Miki N, Ono M, Takano K: Symptomatic hypercalcemia due to painless thyroiditis after unilateral adrenalectomy in a patient with Cushing's syndrome. Inter Med 2008;47:751-756.

4 Bateman A, Singh A, Kral T, Solomon S: The immune-hypothalamic-pituitary-adrenal axis. Endocr Rev 1989;10:92-112.

5 Da Mota F, Murray C, Ezzat S: Overt immune dysfunction after Cushing's syndrome remission: a consecutive case series and review of the literature. J Clin Endocrinol Metab 2011; 96:E1670-E1674.
Desailloud R, Hober D: Viruses and thyroiditis: an update. Virol J 2009;6:5.

7 Cribier B, Caille A, Heid E, Grosshans E: Erythema nodosum and associated diseases: a study of 129 cases. Int J Dermatol 1998;37:667.

8 Elenkov IJ: Glucocorticoids and the Th1/Th2 balance. Ann N Y Acad Sci 2004;1024:138146.

-9 Stojic-Vukanic Z, Rauski A, Kosec D, Radojevic K, Pilipovic I, Leposavic G: Dysregulation of T-cell development in adrenal glucocorticoid-deprived rats. Exp Biol Med 2009; 234:1067-1074. 